\title{
Optimum Erbium Isotopes Composition and Distribution for Power Flattening in Advanced PWR Fuel Assembly
}

\author{
Riham Refeat \\ Nuclear Safety Engineering Department, Nuclear and Radiological Regulatory Authority (NRRA), Nasr City, Cairo 11762, Egypt
}

\begin{abstract}
During long cycles, burnable poisons are used to flatten out the variations in the reactivity, their actual composition and distribution in the fuel assembly can have a significant impact on the power distribution. In this paper, Erbium is used as a burnable poison in a PWR fuel assembly that is burned up to a very high burnup level reaching $70 \mathrm{GWd} / \mathrm{t}$. The optimum composition and distribution of the Erbium isotopes in the assembly are determined to provide reactivity control and obtain flat power distribution. Four models are simulated using MCNPX 2.7 code; the first model used natural Erbium distributed uniformly in all the fuel pins of the assembly, while the other three models used enriched Erbium with different Erbia $\left(\mathrm{Er}_{2} \mathrm{O}_{3}\right)$ concentrations and distributions. The results obtained for k-infinity and pin power peaking factors showed that the suggested loadings of the Erbium bearing pins can sustain very long burnup cycles up to $70 \mathrm{GWd} / \mathrm{t}$. The suggested loading of the Erbia $\left(\operatorname{Er}_{2} \mathrm{O}_{3}\right)$ bearing rods, either in natural or enriched form distributed uniformly in the fuel assembly, can keep the power distribution in the assembly more flattened. Moreover, the peak pin power values are lower compared to the enriched models with partial Erbium distribution.
\end{abstract}

Key words: Erbium burnable poison, PWR assembly, long burnup cycles.

\section{Introduction}

Burnable poisons are used in nuclear power reactors to allow for higher fuel enrichment, which in turn leads to a longer reactor life. They are used to flatten out the variations in reactivity during burnup without the need for a large control rod system. Erbium is one of the major burnable absorber materials used in light water reactor designs. It has also been used in MHTRs (Modular high temperature reactors) and more recently in System-80 PWRs. Erbium is an integral burnable poison blended into the fuel matrix as Erbia $\left(\mathrm{Er}_{2} \mathrm{O}_{3}\right)$. Natural Erbium consists mainly of six isotopes; Er-162, Er-164, Er-166, Er-167, Er-168 and Er-170. The natural Erbium composition is given in Table 1. Er-167 isotope has a large thermal capture cross-section giving an elemental resonance integral of 740 barns [1].

The aim of this paper is to investigate the use of the Erbium burnable poison to provide reactivity control

\footnotetext{
*Corresponding author: Riham Refeat, lecturer, research field: neutronic calculations for nuclear reactors.
}

in a PWR fuel assembly so that it can burn up to a very high level reaching $70 \mathrm{GWd} / \mathrm{t}$. The optimum composition and distribution of the Erbia bearing pins is determined to achieve very long burnup cycles and at the same time flatten the power distribution. The data used for the PWR assembly in this paper are based on the "Reactor Physics Benchmark for LWR Next Generation Fuels”, which was proposed by the Research Committee on Reactor Physics organized by Japan Atomic Energy Research Institute [2]. However in the present work Erbium is used as a burnable poison instead of gadolinium. The MCNPX 2.7 code [3] is used to perform the burnup calculations. The cross section data for most of the isotopes used in the calculations are taken from the ENDFB-VI library (Releases 0 to 6) [4], while the cross section data used for the Erbium isotopes are taken from the recently released nuclear data library ENDFB-VII.1 [5].

\section{PWR Assembly Configuration}

The fuel assembly used in the present paper has the same geometrical configuration as that in the 
Benchmark Problem Suite for Reactor Physics Study of LWR Next Generation Fuels [2]. It is a $17 \times 17$ PWR assembly which is composed of $\mathrm{UO}_{2}$ fuel pins with average U-235 enrichment of about $6.2 \mathrm{wt} \%$ to ensure mean discharge burnup of up to $70 \mathrm{GWd} / \mathrm{t}$ for 21 effective full power month's operation. The assembly contains $24 \mathrm{C} / \mathrm{R}$ guide thimble $(\mathrm{G} / \mathrm{T})$ and one instrumentation guide thimble (I/T). The Gadolinia bearing fuel pins are replaced by Erbia bearing fuel pins $\left(\mathrm{UO}_{2}-\mathrm{Er}_{2} \mathrm{O}_{3}\right)$ with different Erbia concentrations. The concentrations for the natural and enriched models are chosen in order to obtain k-infinity values at zero burnup close to the average value of k-infinity obtained in the LWR next generation fuel benchmark problem [6], this value is taken as a set limit to reduce the computational time.

The geometrical data of the assembly is given in Table 2, while the atomic number densities of the $\mathrm{UO}_{2}$ fuel pins, the structural and moderator materials are

Table 1 The natural erbium composition.

\begin{tabular}{ll}
\hline Isotope & Atom \% \\
\hline Er-162 & 0.14 \\
Er-164 & 1.61 \\
Er-166 & 33.61 \\
Er-167 & 22.93 \\
Er-168 & 26.78 \\
Er-170 & 14.93 \\
\hline
\end{tabular}

Table 2 The geometrical characteristics of the materials.

\begin{tabular}{ll}
\hline Description & $\mathrm{Cm}$ \\
\hline Assembly pitch & 21.505 \\
Fuel rod pitch & 1.265 \\
Pellet outer diameter & 0.824 \\
Cladding inner diameter & 0.824 \\
Cladding outer diameter & 0.952 \\
I/T, G/T inner diameter & 1.14 \\
I/T, G/T outer diameter & 1.22 \\
\hline
\end{tabular}

Table 3 The atomic composition of materials (\#/barn/cm).

\begin{tabular}{lll}
\hline Material & Isotope & Number density \\
\hline \multirow{2}{*}{$\mathrm{UO}_{2}$ Fuel pins } & $\mathrm{U}-235$ & $1.5122 \times 10^{-3}$ \\
& $\mathrm{U}-238$ & $2.1477 \times 10^{-2}$ \\
\multirow{2}{*}{ Structural material } & $\mathrm{O}-16$ & $4.5945 \times 10^{-2}$ \\
\multirow{2}{*}{ Moderator } & $\mathrm{Zr}-\mathrm{nat}$ & $4.3107 \times 10^{-2}$ \\
& $\mathrm{H}-1$ & $4.4161333 \times 10^{-2}$ \\
& $\mathrm{O}-16$ & $2.2080667 \times 10^{-2}$ \\
\hline
\end{tabular}

given in Table 3. The data for the Erbia bearing fuel pins $\left(\mathrm{UO}_{2}-\mathrm{Er}_{2} \mathrm{O}_{3}\right)$ are given in the next section. Burnup calculations are performed under the condition of hot temperature, that is $900(\mathrm{~K})$ for $\mathrm{UO}_{2}$ and $\mathrm{UO}_{2}$ $\mathrm{Er}_{2} \mathrm{O}_{3}$ fuel pins, and $600(\mathrm{~K})$ for structural and moderator materials. The power density is $37.5 \mathrm{~W} / \mathrm{gU}$.

\section{Proposed Assembly Configurations}

Four models are simulated using the MCNPX2.7 code to represent the specified PWR fuel assembly. In the first model (Model A) natural Erbium is used as a burnable poison in the Erbia bearing fuel pins. Erbia is loaded with concentration $1.6 \mathrm{wt} \%$ in all the fuel pins of the assembly to obtain k-infinity value almost equal to the average value obtained in the LWR next generation fuel benchmark problem [6], which is 1.13070. Fig. 1 shows an MCNPX cross sectional view for the natural Erbium model.

In the second model (Model B), enriched Erbium with $100 \%$ Er-167 is used as a burnable poison in the Erbia bearing fuel pin. Three configurations are proposed to obtain k-infinity values almost equal to that of reference (6). In the first configuration (B1), Erbia is loaded in all the fuel pins of the assembly with concentration $0.4 \mathrm{wt} \%$, in the second configuration (B2) Erbia is loaded in 82 fuel rods of the assembly with concentration $1.6 \mathrm{wt} \%$ in $4 \% \mathrm{U}-235$,

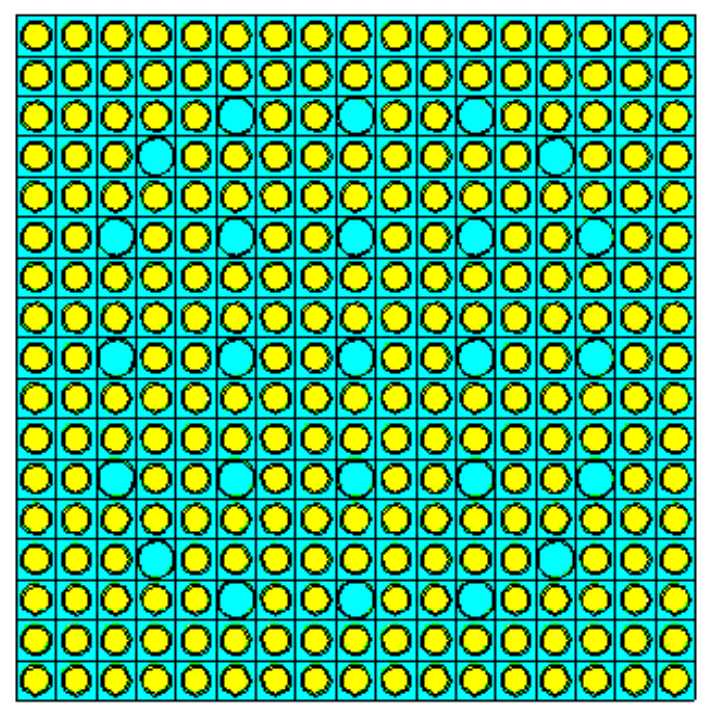

Fig. 1 An MCNPX cross sectional view for the natural Erbium model. 
while in the third configuration (B3) Erbia is loaded in 80 fuel rods of the assembly with concentration 1.7 wt \% in 4\% U-235. Fig. 2 shows an MCNPX cross sectional view for the enriched Erbium models (B2 and B3). The yellow pins represent the Erbia fuel pins.

In all models, the cross section data used for most of the isotopes are taken from the ENDFB-VI library (Releases 0 to 6). The cross section data used for the Erbium isotopes are taken from the recently released nuclear data library ENDFB-VII.1 and are merged with the set of libraries used by the MCNPX 2.7 code. This is done to make use of the latest evaluated Erbium cross section data.

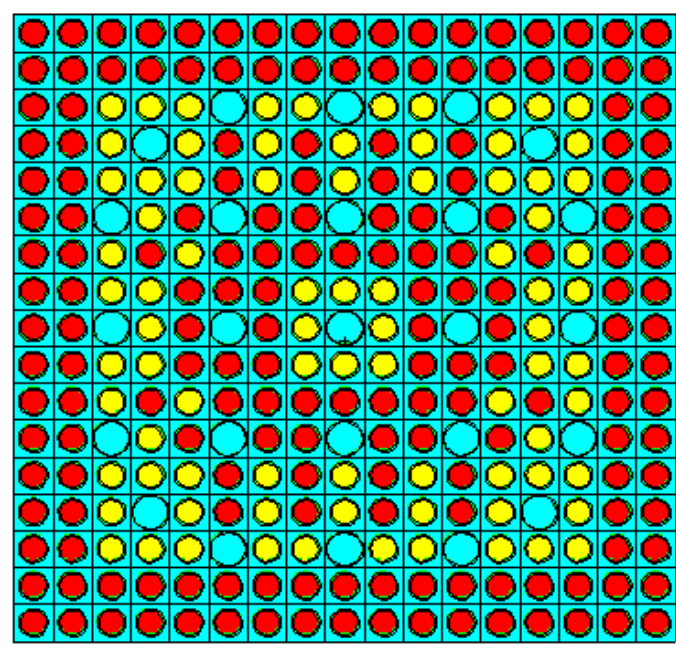

(B2)

\section{Results and Discussion}

\subsection{The Natural Erbium Model}

Fig. 3 shows the values of k-infinity obtained for the natural Erbium model (Model A) at different burnup levels (from 0 to $70 \mathrm{GWd} / \mathrm{t}$ ). The results are compared with those obtained for the same assembly but without the Erbium burnable poison (No Erbium).

The values of k-infinity obtained for Model A increased reaching a maximum value at a burnup 15 GWD/t and then decreased with burnup until it reached its minimum value at $70 \mathrm{GWd} / \mathrm{t}$ in an almost linear manner. Also the figure shows that the addition

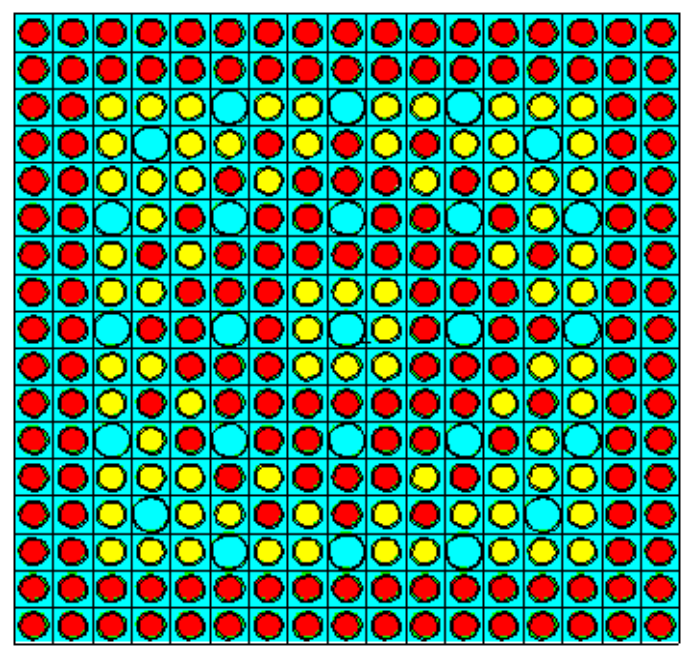

(B3)

Fig. 2 An MCNPX cross sectional view for the enriched erbium models.

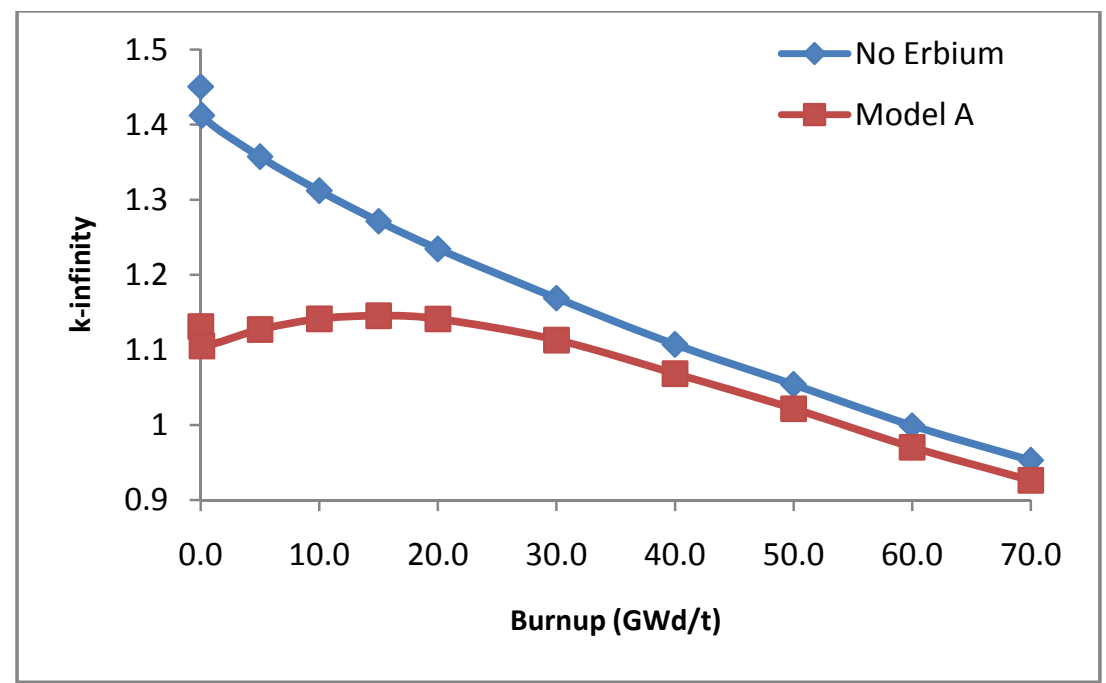

Fig. 3 Comparison of k-infinity between the natural erbium and no erbium models. 
of Erbium tends to decrease the value of k-infinity at 0 GWd/t compared to that without the Erbium burnable poison.

Table 4 shows the values of the pin power peaking factor at the different burnup values obtained for the natural Erbium model compared with those for the No Erbium Model. The values are normalized to one. The estimated relative errors associated with the pin power values are reliable as stated in MCNP manual [3].Tables 5 and 6 present the burnup dependency of the power distributions obtained for both models at burnup value $0 \mathrm{GWd} / \mathrm{t}$ and $70 \mathrm{GWd} / \mathrm{t}$.
Results show that the power distribution in both models is flat. However, it is shown that the addition of Erbium tends to slightly increase the pin power peaking factors.

\subsection{Enriched Erbium Model}

Fig. 4 shows the values of k-infinity obtained for the enriched Erbium models (B1, B2 and B3) at different burnup values (from 0 to $70 \mathrm{GWd} / \mathrm{t}$ ). The results are compared with those obtained for the same assembly but without erbium burnable poison (no erbium).

Table 4 Pin Power Peaking factors for the natural Erbium and no Erbium models.

\begin{tabular}{lll}
\hline Burnup $(\mathrm{GWd} / \mathrm{t})$ & No erbium & Model A \\
\hline 0.0 & $1.086(0.004)$ & $1.093(0.0047)$ \\
0.1 & $1.087(0.0044)$ & $1.092(0.0047)$ \\
5.0 & $1.082(0.0044)$ & $1.090(0.0047)$ \\
10.0 & $1.084(0.0044)$ & $1.091(0.0047)$ \\
15.0 & $1.084(0.0046)$ & $1.086(0.0048)$ \\
20.0 & $1.080(0.0046)$ & $1.080(0.0048)$ \\
30.0 & $1.079(0.0047)$ & $1.079(0.0048)$ \\
40.0 & $1.067(0.0047)$ & $1.066(0.0048)$ \\
50.0 & $1.062(0.0047)$ & $1.061(0.0048)$ \\
60.0 & $1.062(0.0047)$ & $1.067(0.0048)$ \\
70.0 & $1.066(0.0047)$ & $1.065(0.0048)$ \\
\hline
\end{tabular}

Table 5 Pin power distribution at $0 \mathrm{GWd} / \mathrm{t}$ for the natural erbium and no erbium models.

\begin{tabular}{|c|c|c|c|c|c|c|c|c|c|}
\hline & 0.000 & $\begin{array}{l}1.065 \\
1.068\end{array}$ & $\begin{array}{l}1.068 \\
1.068\end{array}$ & 0.000 & $\begin{array}{l}1.055 \\
1.060\end{array}$ & $\begin{array}{l}1.056 \\
1.054\end{array}$ & 0.000 & $\begin{array}{l}1.016 \\
1.030\end{array}$ & $\begin{array}{l}0.950 \\
0.956\end{array}$ \\
\hline \multirow{16}{*}{$\begin{array}{l}\text { No Erbium } \\
\text { Model A }\end{array}$} & 1.065 & 1.044 & 1.025 & 1.057 & 1.022 & 1.011 & 1.043 & 0.980 & 0.945 \\
\hline & 1.068 & 1.030 & 1.027 & 1.069 & 1.024 & 1.018 & 1.041 & 0.982 & 0.950 \\
\hline & 1.068 & 1.025 & 1.024 & 1.061 & 1.021 & 1.016 & 1.047 & 0.983 & 0.949 \\
\hline & 1.068 & 1.027 & 1.026 & 1.069 & 1.026 & 1.017 & 1.047 & 0.981 & 0.942 \\
\hline & & 1.057 & 1.061 & & 1.066 & 1.066 & & 1.016 & 0.952 \\
\hline & 0.000 & 1.069 & 1.069 & 0.000 & 1.075 & 1.070 & 0.000 & 1.019 & 0.946 \\
\hline & 1.055 & 1.022 & 1.021 & 1.066 & 1.051 & 1.086 & 1.060 & 0.975 & 0.938 \\
\hline & 1.060 & 1.024 & 1.026 & 1.075 & 1.059 & 1.093 & 1.064 & 0.976 & 0.937 \\
\hline & 1.056 & 1.011 & 1.016 & 1.066 & 1.086 & & 1.032 & 0.946 & 0.923 \\
\hline & 1.054 & 1.018 & 1.017 & 1.070 & 1.093 & 0.000 & 1.032 & 0.951 & 0.918 \\
\hline & & 1.043 & 1.047 & & 1.060 & 1.032 & 0.964 & 0.925 & 0.909 \\
\hline & 0.000 & 1.041 & 1.047 & 0.000 & 1.064 & 1.032 & 0.959 & 0.923 & 0.911 \\
\hline & 1.016 & 0.980 & 0.983 & 1.016 & 0.975 & 0.946 & 0.925 & 0.909 & 0.900 \\
\hline & 1.030 & 0.982 & 0.981 & 1.019 & 0.976 & 0.951 & 0.923 & 0.909 & 0.892 \\
\hline & 0.950 & 0.945 & 0.949 & 0.952 & 0.938 & 0.923 & 0.909 & 0.900 & 0.893 \\
\hline & 0.956 & 0.950 & 0.942 & 0.946 & 0.937 & 0.918 & 0.911 & 0.892 & 0.886 \\
\hline
\end{tabular}


Table 6 Pin power distribution at $70 \mathrm{GWd} / \mathrm{t}$ for the natural erbium and no erbium models.

\begin{tabular}{|c|c|c|c|c|c|c|c|c|c|}
\hline & 0.000 & $\begin{array}{l}1.035 \\
1.031 \\
\end{array}$ & $\begin{array}{l}1.046 \\
1.049\end{array}$ & 0.000 & $\begin{array}{l}1.033 \\
1.028 \\
\end{array}$ & $\begin{array}{l}1.033 \\
1.017 \\
\end{array}$ & 0.000 & $\begin{array}{l}1.055 \\
1.065\end{array}$ & $\begin{array}{l}0.987 \\
0.988\end{array}$ \\
\hline \multirow{16}{*}{$\begin{array}{l}\text { No Erbium } \\
\text { Model A }\end{array}$} & 1.035 & 1.007 & 1.000 & 1.040 & 0.999 & 0.988 & 1.012 & 1.024 & 0.977 \\
\hline & 1.031 & 1.002 & 0.998 & 1.044 & 0.994 & 0.979 & 1.010 & 1.029 & 0.987 \\
\hline & 1.046 & 1.000 & 1.008 & 1.043 & 1.024 & 0.988 & 1.000 & 1.030 & 0.981 \\
\hline & 1.049 & 0.998 & 1.006 & 1.044 & 1.020 & 0.989 & 1.001 & 1.027 & 0.977 \\
\hline & & 1.040 & 1.043 & & 1.039 & 1.027 & & 1.066 & 0.982 \\
\hline & 0.000 & 1.044 & 1.044 & 0.000 & 1.044 & 1.032 & 0.000 & 1.065 & 0.982 \\
\hline & 1.033 & 0.999 & 1.024 & 1.039 & 1.026 & 1.045 & 1.026 & 1.018 & 0.969 \\
\hline & 1.028 & 0.994 & 1.020 & 1.044 & 1.028 & 1.042 & 1.022 & 1.021 & 0.963 \\
\hline & 1.023 & 0.988 & 0.988 & 1.027 & 1.045 & & 0.980 & 0.987 & 0.955 \\
\hline & 1.017 & 0.979 & 0.989 & 1.032 & 1.042 & 0.000 & 0.983 & 0.984 & 0.952 \\
\hline & & 1.012 & 1.000 & & 1.026 & 0.980 & 0.928 & 0.961 & 0.932 \\
\hline & 0.000 & 1.010 & 1.001 & 0.000 & 1.022 & 0.983 & 0.931 & 0.963 & 0.933 \\
\hline & 1.055 & 1.024 & 1.030 & 1.066 & 1.018 & 0.987 & 0.961 & 0.932 & 0.921 \\
\hline & 1.065 & 1.029 & 1.027 & 1.065 & 1.021 & 0.984 & 0.963 & 0.937 & 0.918 \\
\hline & 0.987 & 0.977 & 0.981 & 0.982 & 0.969 & 0.955 & 0.932 & 0.921 & 0.917 \\
\hline & 0.988 & 0.987 & 0.977 & 0.982 & 0.963 & 0.952 & 0.933 & 0.918 & 0.907 \\
\hline
\end{tabular}

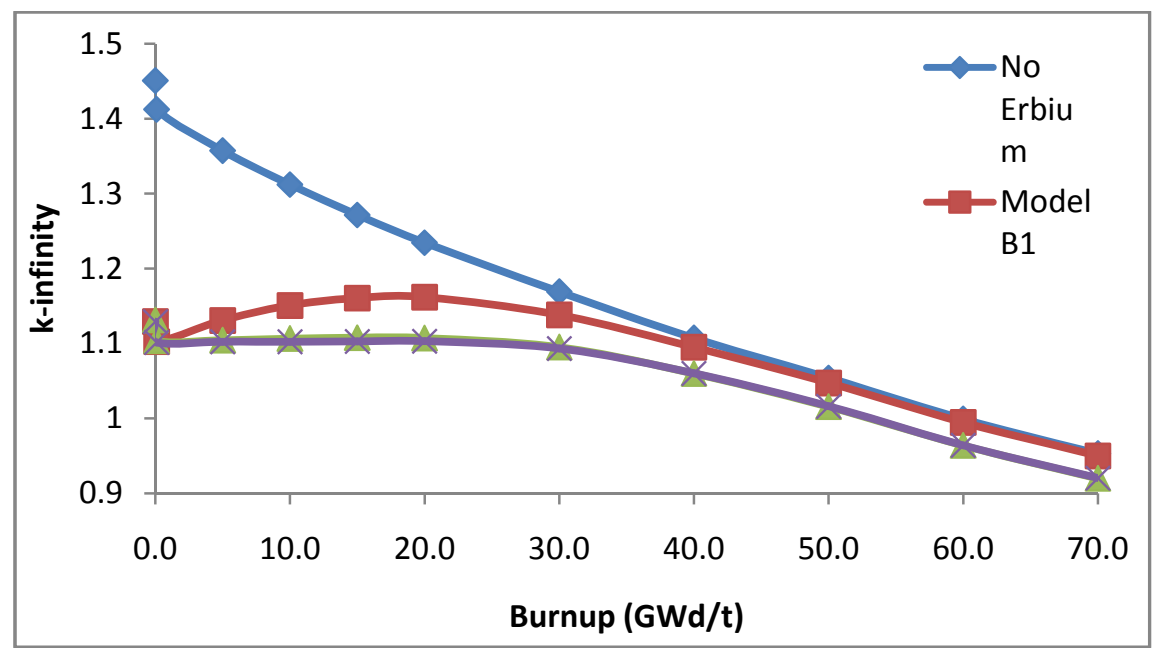

Fig. 4 Comparison of k-infinity between the enriched erbium and no erbium models.

Similarly as in the natural Erbium model, the addition of Erbium tends to decrease the value of k-infinity at $0 \mathrm{GWd} / \mathrm{t}$. Also the figure shows that the values of k-infinity for the three concentrations increased slightly reaching a maximum value and then decreased with burnup until it reached its minimum value at $70 \mathrm{GWd} / \mathrm{t}$ in an almost linear manner. However the maximum value obtained for model B1 is higher than those obtained for models B2 and B3 and is closer to that obtained in the natural Erbium model.

The values of the pin power peaking factors obtained for the enriched Erbium models compared to those obtained for the no Erbium model at the different burnup values are shown in Table 7. The values are normalized to one. The estimated relative errors associated with the pin power values for the enriched Erbium models are reliable as stated in MCNP manual [3]. Tables 8-13 present the burnup dependency of the power distributions obtained at burnup value $0 \mathrm{GWd} / \mathrm{t}$ and $70 \mathrm{GWd} / \mathrm{t}$ for the Erbia (0.4 wt \%), Erbia (1.6wt \%) and Erbia (1.7wt \%) respectively.

It is shown that the addition of Erbium tends to increase the pin power peaking factors. However, the 
Table 7 Pin Power Peaking factors for the enriched Erbium and no Erbium models.

\begin{tabular}{lllll}
\hline Burnup (GWd/t) & No Erbium & Model B1 & Model B2 & Model B3 \\
\hline 0.0 & $1.086(0.004)$ & $1.093(0.0047)$ & $1.217(0.0045)$ & $1.222(0.0045)$ \\
0.1 & $1.087(0.0044)$ & $1.099(0.0047)$ & $1.215(0.0045)$ & $1.228(0.0045)$ \\
5.0 & $1.082(0.0044)$ & $1.091(0.0047)$ & $1.218(0.0045)$ & $1.197(0.0045)$ \\
10.0 & $1.084(0.0044)$ & $1.095(0.0047)$ & $1.188(0.0045)$ & $1.197(0.0045)$ \\
15.0 & $1.084(0.0046)$ & $1.090(0.0047)$ & $1.175(0.0045)$ & $1.171(0.0045)$ \\
20.0 & $1.080(0.0046)$ & $1.087(0.0047)$ & $1.169(0.0047)$ & $1.168(0.0045)$ \\
30.0 & $1.079(0.0047)$ & $1.084(0.0047)$ & $1.160(0.0047)$ & $1.160(0.0047)$ \\
40.0 & $1.067(0.0047)$ & $1.076(0.0048)$ & $1.141(0.0047)$ & $1.143(0.0047)$ \\
50.0 & $1.062(0.0047)$ & $1.076(0.0048)$ & $1.117(0.0047)$ & $1.135(0.0047)$ \\
60.0 & $1.062(0.0047)$ & $1.075(0.0048)$ & $1.111(0.0047)$ & $1.113(0.0048)$ \\
70.0 & $1.066(0.0047)$ & $1.078(0.0048)$ & $1.110(0.0047)$ & $1.110(0.0048)$ \\
\hline
\end{tabular}

Table 8 Pin power distribution at $0 \mathrm{GWd} / \mathrm{t}$ for model B1 and no erbium.

\begin{tabular}{|c|c|c|c|c|c|c|c|c|c|}
\hline & 0.000 & $\begin{array}{l}1.065 \\
1.060 \\
\end{array}$ & $\begin{array}{l}1.068 \\
1.056 \\
\end{array}$ & 0.000 & $\begin{array}{l}1.055 \\
1.061 \\
\end{array}$ & $\begin{array}{l}1.056 \\
1.050 \\
\end{array}$ & 0.000 & \begin{tabular}{|l|}
1.016 \\
1.016 \\
\end{tabular} & $\begin{array}{l}0.950 \\
0.940 \\
\end{array}$ \\
\hline \multirow{16}{*}{$\begin{array}{l}\text { No Erbium } \\
\text { Model B1 }\end{array}$} & 1.065 & 1.044 & 1.025 & 1.057 & 1.022 & 1.011 & 1.043 & 0.980 & 0.945 \\
\hline & 1.060 & 1.026 & 1.016 & 1.067 & 1.029 & 1.034 & 1.033 & 0.970 & 0.954 \\
\hline & 1.068 & 1.025 & 1.024 & 1.061 & 1.021 & 1.016 & 1.047 & 0.983 & 0.949 \\
\hline & 1.056 & 1.016 & 1.019 & 1.058 & 1.015 & 1.029 & 1.051 & 0.991 & 0.951 \\
\hline & & 1.057 & 1.061 & & 1.066 & 1.066 & & 1.016 & 0.952 \\
\hline & 0.000 & 1.067 & 1.058 & 0.000 & 1.079 & 1.074 & 0.000 & 1.038 & 0.957 \\
\hline & 1.055 & 1.022 & 1.021 & 1.066 & 1.051 & 1.086 & 1.060 & 0.975 & 0.938 \\
\hline & 1.061 & 1.029 & 1.015 & 1.079 & 1.056 & 1.093 & 1.066 & 0.989 & 0.937 \\
\hline & 1.056 & 1.011 & 1.016 & 1.066 & 1.086 & & 1.032 & 0.946 & 0.923 \\
\hline & 1.050 & 1.034 & 1.029 & 1.074 & 1.093 & 0.000 & 1.041 & 0.954 & 0.940 \\
\hline & & 1.043 & 1.047 & & 1.060 & 1.032 & 0.964 & 0.925 & 0.909 \\
\hline & 0.000 & 1.033 & 1.051 & 0.000 & 1.066 & 1.041 & 0.965 & 0.927 & 0.910 \\
\hline & 1.016 & 0.980 & 0.983 & 1.016 & 0.975 & 0.946 & 0.925 & 0.909 & 0.900 \\
\hline & 1.016 & 0.970 & 0.991 & 1.038 & 0.989 & 0.954 & 0.927 & 0.908 & 0.880 \\
\hline & 0.950 & 0.945 & 0.949 & 0.952 & 0.938 & 0.923 & 0.909 & 0.900 & 0.893 \\
\hline & 0.940 & 0.954 & 0.951 & 0.957 & 0.937 & 0.940 & 0.910 & 0.880 & 0.887 \\
\hline
\end{tabular}

Table 9 Pin power distribution at $70 \mathrm{GWd} / \mathrm{t}$ for model B1 and no erbium.

\begin{tabular}{|c|c|c|c|c|c|c|c|c|c|}
\hline & 0.000 & $\begin{array}{l}1.035 \\
1.035\end{array}$ & $\begin{array}{l}1.046 \\
1.049 \\
\end{array}$ & 0.000 & $\begin{array}{l}1.033 \\
1.026\end{array}$ & $\begin{array}{l}1.033 \\
1.015 \\
\end{array}$ & 0.000 & $\begin{array}{l}1.055 \\
1.057\end{array}$ & $\begin{array}{l}0.987 \\
0.991\end{array}$ \\
\hline \multirow{16}{*}{$\begin{array}{l}\text { No Erbium } \\
\text { Model B1 }\end{array}$} & 1.035 & 1.007 & 1.000 & 1.040 & 0.999 & 0.988 & 1.012 & 1.024 & 0.977 \\
\hline & 1.035 & 1.012 & 1.018 & 1.033 & 1.005 & 0.990 & 1.024 & 1.039 & 0.987 \\
\hline & 1.046 & 1.000 & 1.008 & 1.043 & 1.024 & 0.988 & 1.000 & 1.030 & 0.981 \\
\hline & 1.049 & 1.018 & 0.995 & 1.031 & 1.013 & 0.995 & 1.010 & 1.042 & 0.987 \\
\hline & & 1.040 & 1.043 & & 1.039 & 1.027 & & 1.066 & 0.982 \\
\hline & 0.000 & 1.033 & 1.031 & 0.000 & 1.035 & 1.024 & 0.000 & 1.078 & 1.003 \\
\hline & 1.033 & 0.999 & 1.024 & 1.039 & 1.026 & 1.045 & 1.026 & 1.018 & 0.969 \\
\hline & 1.026 & 1.005 & 1.013 & 1.035 & 1.009 & 1.053 & 1.037 & 1.023 & 0.964 \\
\hline & 1.023 & 0.988 & 0.988 & 1.027 & 1.045 & & 0.980 & 0.987 & 0.955 \\
\hline & 1.015 & 0.990 & 0.995 & 1.024 & 1.053 & 0.000 & 0.980 & 1.001 & 0.966 \\
\hline & & 1.012 & 1.000 & & 1.026 & 0.980 & 0.928 & 0.961 & 0.932 \\
\hline & 0.000 & 1.024 & 1.010 & 0.000 & 1.037 & 0.980 & 0.934 & 0.953 & 0.945 \\
\hline & 1.055 & 1.024 & 1.030 & 1.066 & 1.018 & 0.987 & 0.961 & 0.932 & 0.921 \\
\hline & 1.057 & 1.039 & 1.042 & 1.078 & 1.023 & 1.001 & 0.953 & 0.941 & 0.920 \\
\hline & 0.987 & 0.977 & 0.981 & 0.982 & 0.969 & 0.955 & 0.932 & 0.921 & 0.917 \\
\hline & 0.991 & 0.987 & 0.987 & 1.003 & 0.964 & 0.966 & 0.945 & 0.920 & 0.916 \\
\hline
\end{tabular}



Advanced PWR Fuel Assembly

Table 10 Pin power distribution at 0 GWd/t for model B2 and no erbium.

\begin{tabular}{|c|c|c|c|c|c|c|c|c|c|}
\hline \multirow{17}{*}{$\begin{array}{l}\text { No Erbium } \\
\text { Model B2 }\end{array}$} & 0.000 & $\begin{array}{l}1.065 \\
0.685\end{array}$ & $\begin{array}{l}1.068 \\
1.184\end{array}$ & 0.000 & $\begin{array}{l}1.055 \\
1.187\end{array}$ & $\begin{array}{l}1.056 \\
0.686\end{array}$ & 0.000 & $\begin{array}{l}1.016 \\
1.179\end{array}$ & $\begin{array}{l}0.950 \\
1.131\end{array}$ \\
\hline & 1.065 & 1.044 & 1.025 & 1.057 & 1.022 & 1.011 & 1.043 & 0.980 & 0.945 \\
\hline & 0.685 & 0.674 & 1.157 & 1.217 & 1.165 & 0.652 & 0.689 & 1.148 & 1.127 \\
\hline & 1.068 & 1.025 & 1.024 & 1.061 & 1.021 & 1.016 & 1.047 & 0.983 & 0.949 \\
\hline & 1.184 & 1.157 & 1.170 & 1.202 & 0.674 & 1.099 & 0.680 & 1.140 & 1.125 \\
\hline & & 1.057 & 1.061 & & 1.066 & 1.066 & & 1.016 & 0.952 \\
\hline & 0.000 & 1.217 & 1.202 & 0.000 & 1.154 & 0.691 & 0.000 & 1.178 & 1.118 \\
\hline & 1.055 & 1.022 & 1.021 & 1.066 & 1.051 & 1.086 & 1.060 & 0.975 & 0.938 \\
\hline & 0.708 & 1.165 & 0.674 & 1.154 & 0.674 & 0.682 & 0.687 & 1.137 & 1.108 \\
\hline & 1.056 & 1.011 & 1.016 & 1.066 & 1.086 & & 1.032 & 0.946 & 0.923 \\
\hline & 0.686 & 1.106 & 0.657 & 1.180 & 0.682 & 0.000 & 0.674 & 1.085 & 1.109 \\
\hline & & 1.043 & 1.047 & & 1.060 & 1.032 & 0.964 & 0.925 & 0.909 \\
\hline & 0.000 & 0.689 & 0.680 & 0.000 & 0.687 & 0.674 & 0.648 & 1.115 & 1.108 \\
\hline & 1.016 & 0.980 & 0.983 & 1.016 & 0.975 & 0.946 & 0.925 & 0.909 & 0.900 \\
\hline & 1.179 & 1.148 & 1.140 & 1.178 & 1.137 & 1.085 & 1.115 & 1.099 & 1.114 \\
\hline & 0.950 & 0.945 & 0.949 & 0.952 & 0.938 & 0.923 & 0.909 & 0.900 & 0.893 \\
\hline & 1.131 & 1.127 & 1.125 & 1.118 & 1.108 & 1.109 & 1.108 & 1.114 & 1.117 \\
\hline
\end{tabular}

Table 11 Pin power distribution at $70 \mathrm{GWd} / \mathrm{t}$ for model $\mathrm{B} 2$ and no erbium.

\begin{tabular}{|c|c|c|c|c|c|c|c|c|c|}
\hline & 0.000 & $\begin{array}{l}1.035 \\
0.967\end{array}$ & $\begin{array}{l}1.046 \\
1.077\end{array}$ & 0.000 & $\begin{array}{l}1.033 \\
1.063\end{array}$ & $\begin{array}{l}1.033 \\
0.963\end{array}$ & 0.000 & $\begin{array}{l}1.055 \\
1.110\end{array}$ & $\begin{array}{l}0.987 \\
1.026\end{array}$ \\
\hline No Erbium & 1.035 & 1.007 & 1.000 & 1.040 & 0.999 & 0.988 & 1.012 & 1.024 & 0.977 \\
\hline \multirow[t]{15}{*}{ Model B2 } & 0.967 & 0.953 & 1.041 & 1.056 & 1.036 & 0.923 & 0.938 & 1.083 & 0.999 \\
\hline & 1.046 & 1.000 & 1.008 & 1.043 & 1.024 & 0.988 & 1.000 & 1.030 & 0.981 \\
\hline & 1.077 & 1.041 & 1.029 & 1.053 & 0.921 & 1.019 & 0.926 & 1.084 & 1.012 \\
\hline & & 1.040 & 1.043 & & 1.039 & 1.027 & & 1.066 & 0.982 \\
\hline & 0.000 & 1.056 & 1.053 & 0.000 & 1.084 & 0.977 & 0.000 & 1.095 & 0.999 \\
\hline & 1.033 & 0.999 & 1.024 & 1.039 & 1.026 & 1.045 & 1.026 & 1.018 & 0.969 \\
\hline & 0.967 & 1.036 & 0.921 & 1.084 & 0.943 & 0.962 & 0.935 & 1.049 & 0.981 \\
\hline & 1.023 & 0.988 & 0.988 & 1.027 & 1.045 & & 0.980 & 0.987 & 0.955 \\
\hline & 0.963 & 1.024 & 0.920 & 1.051 & 0.962 & 0.000 & 0.900 & 1.014 & 0.976 \\
\hline & & 1.012 & 1.000 & & 1.026 & 0.980 & 0.928 & 0.961 & 0.932 \\
\hline & 0.000 & 0.938 & 0.926 & 0.000 & 0.935 & 0.900 & 0.854 & 0.979 & 0.953 \\
\hline & 1.055 & 1.024 & 1.030 & 1.066 & 1.018 & 0.987 & 0.961 & 0.932 & 0.921 \\
\hline & 1.110 & 1.083 & 1.084 & 1.095 & 1.049 & 1.014 & 0.979 & 0.972 & 0.937 \\
\hline & 0.987 & 0.977 & 0.981 & 0.982 & 0.969 & 0.955 & 0.932 & 0.921 & 0.917 \\
\hline & 1.026 & 0.999 & 1.012 & 0.999 & 0.981 & 0.976 & 0.953 & 0.937 & 0.945 \\
\hline
\end{tabular}

values of the pin power peaking factors for B2 and B3 models are higher than those obtained in the natural Erbium model or B1 model. The results obtained for the pin power distribution show that it is more flattened in model B1 than those in B2 and B3. This is because the distribution of Erbia bearing fuel pins in models B2 and B3 is in a number of pins unlike the distribution in the natural Erbium model or B1 model, where the Erbia bearing fuel pins are distributed uniformly in all the fuel pins. 
Table 12 Pin power distribution at $0 \mathrm{GWd} / \mathrm{t}$ for model B3 and no erbium.

\begin{tabular}{|c|c|c|c|c|c|c|c|c|c|}
\hline & 0.000 & $\begin{array}{l}1.065 \\
0.684\end{array}$ & $\begin{array}{l}1.068 \\
1.205\end{array}$ & 0.000 & $\begin{array}{l}1.055 \\
1.208\end{array}$ & $\begin{array}{l}1.056 \\
1.166\end{array}$ & 0.000 & $\begin{array}{l}1.016 \\
1.194 \\
\end{array}$ & $\begin{array}{l}0.950 \\
1.152\end{array}$ \\
\hline \multirow{16}{*}{$\begin{array}{l}\text { No Erbium } \\
\text { Model B3 }\end{array}$} & 1.065 & 1.044 & 1.025 & 1.057 & 1.022 & 1.011 & 1.043 & 0.980 & 0.945 \\
\hline & 0.684 & 0.669 & 1.145 & 1.222 & 1.151 & 0.671 & 0.689 & 1.130 & 1.124 \\
\hline & 1.068 & 1.025 & 1.024 & 1.061 & 1.021 & 1.016 & 1.047 & 0.983 & 0.949 \\
\hline & 1.205 & 1.145 & 1.170 & 1.195 & 0.672 & 1.104 & 0.675 & 1.137 & 1.136 \\
\hline & & 1.057 & 1.061 & & 1.066 & 1.066 & & 1.016 & 0.952 \\
\hline & 0.000 & 1.222 & 1.195 & 0.000 & 1.169 & 0.672 & 0.000 & 1.190 & 1.143 \\
\hline & 1.055 & 1.022 & 1.021 & 1.066 & 1.051 & 1.086 & 1.060 & 0.975 & 0.938 \\
\hline & 1.208 & 1.151 & 0.672 & 1.169 & 0.656 & 0.669 & 0.688 & 1.135 & 1.142 \\
\hline & 1.056 & 1.011 & 1.016 & 1.066 & 1.086 & & 1.032 & 0.946 & 0.923 \\
\hline & 1.166 & 0.671 & 1.104 & 0.672 & 0.669 & 0.000 & 0.659 & 1.112 & 1.108 \\
\hline & & 1.043 & 1.047 & & 1.060 & 1.032 & 0.964 & 0.925 & 0.909 \\
\hline & 0.000 & 0.689 & 0.675 & 0.000 & 0.688 & 0.659 & 0.656 & 1.098 & 1.108 \\
\hline & 1.016 & 0.980 & 0.983 & 1.016 & 0.975 & 0.946 & 0.925 & 0.909 & 0.900 \\
\hline & 1.194 & 1.130 & 1.137 & 1.190 & 1.135 & 1.112 & 1.098 & 1.116 & 1.116 \\
\hline & 0.950 & 0.945 & 0.949 & 0.952 & 0.938 & 0.923 & 0.909 & 0.900 & 0.893 \\
\hline & 1.152 & 1.124 & 1.136 & 1.143 & 1.142 & 1.108 & 1.108 & 1.116 & 1.119 \\
\hline
\end{tabular}

Table 13 Pin power distribution at $70 \mathrm{GWd} / \mathrm{t}$ for model $\mathrm{B} 3$ and no erbium.

\begin{tabular}{|c|c|c|c|c|c|c|c|c|c|}
\hline & 0.000 & $\begin{array}{l}1.035 \\
0.947\end{array}$ & $\begin{array}{l}1.046 \\
1.074\end{array}$ & 0.000 & $\begin{array}{l}1.033 \\
1.052 \\
\end{array}$ & $\begin{array}{l}1.033 \\
1.055 \\
\end{array}$ & 0.000 & $\begin{array}{l}1.055 \\
1.110 \\
\end{array}$ & $\begin{array}{l}0.987 \\
1.022 \\
\end{array}$ \\
\hline \multirow{16}{*}{$\begin{array}{l}\text { No Erbium } \\
\text { Model B3 }\end{array}$} & 1.035 & 1.007 & 1.000 & 1.040 & 0.999 & 0.988 & 1.012 & 1.024 & 0.977 \\
\hline & 0.947 & 0.944 & 1.034 & 1.038 & 1.028 & 0.938 & 0.927 & 1.065 & 1.008 \\
\hline & 1.046 & 1.000 & 1.008 & 1.043 & 1.024 & 0.988 & 1.000 & 1.030 & 0.981 \\
\hline & 1.074 & 1.034 & 1.034 & 1.067 & 0.945 & 1.036 & 0.929 & 1.058 & 1.012 \\
\hline & & 1.040 & 1.043 & & 1.039 & 1.027 & & 1.066 & 0.982 \\
\hline & 0.000 & 1.038 & 1.067 & 0.000 & 1.098 & 0.973 & 0.000 & 1.086 & 1.015 \\
\hline & 1.033 & 0.999 & 1.024 & 1.039 & 1.026 & 1.045 & 1.026 & 1.018 & 0.969 \\
\hline & 1.052 & 1.028 & 0.945 & 1.098 & 0.959 & 0.971 & 0.925 & 1.033 & 0.998 \\
\hline & 1.023 & 0.988 & 0.988 & 1.027 & 1.045 & & 0.980 & 0.987 & 0.955 \\
\hline & 1.055 & 0.938 & 1.036 & 0.973 & 0.971 & 0.000 & 0.915 & 1.016 & 0.972 \\
\hline & & 1.012 & 1.000 & & 1.026 & 0.980 & 0.928 & 0.961 & 0.932 \\
\hline & 0.000 & 0.927 & 0.929 & 0.000 & 0.925 & 0.915 & 0.860 & 0.994 & 0.952 \\
\hline & 1.055 & 1.024 & 1.030 & 1.066 & 1.018 & 0.987 & 0.961 & 0.932 & 0.921 \\
\hline & 1.110 & 1.065 & 1.058 & 1.086 & 1.033 & 1.016 & 0.994 & 0.966 & 0.939 \\
\hline & 0.987 & 0.977 & 0.981 & 0.982 & 0.969 & 0.955 & 0.932 & 0.921 & 0.917 \\
\hline & 1.022 & 1.008 & 1.012 & 1.015 & 0.998 & 0.972 & 0.952 & 0.939 & 0.938 \\
\hline
\end{tabular}

\section{Conclusions}

Four models were simulated using MCNPX 2.7 code for a $17 \times 17$ PWR fuel assembly with $\mathrm{UO}_{2}$ fuel enriched to $6.2 \% \mathrm{U}-235$ and with burnable poison Erbium oxide $\left(\mathrm{Er}_{2} \mathrm{O}_{3}\right)$. The assembly was assumed to burn up to a very high burnup level reaching 70 GWd/t. The first model used natural Erbium as a burnable poison distributed in all the fuel pins of the assembly, while the other three models used enriched Erbium with different concentrations and distribution. Values of k-infinity and pin power distribution were calculated for all models at the different burnup steps.

It was found that for the all models the values of k-infinity increased reaching a maximum value then decreased with burnup until it reached its minimum 


\section{Optimum Erbium Isotopes Composition and Distribution for Power Flattening in Advanced PWR Fuel Assembly}

value at $70 \mathrm{GWd} / \mathrm{t}$ in an almost linear manner. However the maximum value obtained for the natural Erbium model and the enriched Erbium model with Erbia uniformly distributed in all the fuel pins of the assembly is higher than those obtained for the models with partial distribution of Erbia. Results obtained for the pin power distribution in models with full uniform distribution of Erbia are more flattened and the values of the peak pin power values are minimized compared to those of the models with partial distribution of Erbia.

The present work has shown that the Erbium burnable poison can be used to provide reactivity control in a PWR fuel assembly which burn at very high burnup reaching $70 \mathrm{GWd} / \mathrm{t}$. The suggested loading of the Erbia $\left(\mathrm{Er}_{2} \mathrm{O}_{3}\right)$ bearing rods uniformly distributed in the whole fuel assembly pins, either in natural or enriched form, is optimum to achieve very long burnup cycles and at the same time keeps the power distribution as flat as possible. Also, it has been demonstrated that using enriched Erbium (100\% Er-167) decreased the amount of Erbia needed in case of full distribution from $1.6 \%$ to $0.4 \%$. On the other hand, the peak pin power values obtained for the enriched model with partial Erbia distribution are higher.

\section{References}

[1] Cacuci, Dan G. 2010. Handbook of Nuclear Engineering. Volume I. Springer Science + Business Media.

[2] Yamamoto, A., Ikehara, T., Ito, T. and Saji, E. 2002. "Benchmark Problem Suite for Reactor Physics Study of LWR Next Generation Fuels." Journal of Nuclear Science and Technology 39 (8): 900-12.

[3] Pelowitz, D. B. 2011. MCNPX User's Manual Version 2.7.0. Los Alamos National Laboratory Report LA-CP-11-00438.

[4] Chadwick, M. B. et al. 2006. "Next Generation Evaluated Nuclear Data Library for Nuclear Science and Technology.” Nuclear Data Sheets 107.

[5] Chadwick, M. B. et al. 2011. "Nuclear Data for Science and Technology: Cross Sections, Covariance, Fission Product Yields and Decay Data.” Nuclear Data Sheets 112: 2887-996.

[6] Working Party (WP) website, Japan Atomic Energy Research Institute (JAERI). 1999. "UO ${ }_{2}$ PWR assembly Results.” Accessed 2014. http://hachi.tokai.jaeri.go.jp/Committee/LWR-benchmark/. 